\title{
PROSPECÇÃO DE PATENTES RELACIONADAS COM MARCADORES DE COMBUSTÍVEIS LÍQUIDOS
}

\author{
Edvaldo Pereira Queiroz Júnior ; Leonardo Sena Gomes Teixeira; Sílvio do Desterro Cunha \\ Instituto de Química, Universidade Federal da Bahia (aldo130263@gmail.com)
}

\section{RESUMO}

O trabalho a seguir consiste na apresentação da pesquisa de prospecção de marcadores utilizados em combustíveis líquidos, principalmente de automotores, considerando as diversas aplicações destas substâncias marcadoras e os tipos de métodos de análise e identificação de adulteração de combustíveis. A marcação de combustíveis no Brasil é aplicada para a personalização do produto, como identificação do fabricante ou fornecedor (distribuidor); como também, no intuito de identificação de adulteração desses combustíveis - um problema que ainda atinge todo o país e traz muitos prejuízos econômicos, tanto para o consumidor como para a União.

Palavras Chave: combustível líquido; marcador; adulteração.

\begin{abstract}
The following work is the presentation of research survey of the markers used in liquid fuels, mainly automotive, considering the various applications of these marker substances and the types of methods of analysis and identification of fuel adulteration. The marking of fuels in Brazil is applied to the product customization, such as identification of the manufacturer or supplier (distributor), but also in order to identify adulteration of these - a problem that still affects the whole country and has many economic losses, both for the consumer as to the Union
\end{abstract}

Key words: liquid fuel; marker; adulteration.

Área tecnológica: Energia (Fontes Tradicionais); Bioderivados (combustíveis e químicos) 


\section{INTRODUÇÃO}

O problema de adulteração de combustíveis no Brasil se alastra por todo o país trazendo conseqüências desastrosas econômicas, tanto para o consumidor final como para os cofres da União.

As atividades de monitoramento da qualidade dos combustíveis no Brasil iniciaram-se no $2^{\circ}$ semestre de 1999, com o Programa de Monitoramento da Qualidade dos Combustíveis, da ANP, Agência Nacional do Petróleo, Gás Natural e Biocombustíveis (ANP, n. 248 e 311, 2000). Com a implementação desse programa, iniciou-se um processo de maior controle sobre a distribuição e comercialização dos combustíveis, contra um crescente movimento de adulteração e fraudes que causam prejuízos a União e as secretarias estaduais da fazenda, por perda de arrecadação, acarretando num grande prejuízo econômico-social para o país.

A adulteração dos combustíveis, além de lesar diretamente o consumidor final por estar adquirindo um produto não controlado, que pode estar fora de especificação ou de qualidade duvidosa, geralmente provoca prejuízos econômicos, relacionados ao menor desempenho do combustível e/ou problemas com o motor e manutenção desse, como também, ambientais (WIEDMANN, 2005).

Apesar da ampliação do programa do monitoramento, que hoje cobre praticamente todo o território nacional, cada vez mais se sofisticam as formas de adulteração e de ilicitudes na comercialização dos combustíveis. A ANP divulga, através do seu boletim mensal na página da internet, os dados obtidos nesse programa de monitoramento. Mesmo havendo uma redução nos índices de não conformidades, com o passar dos anos, devido, principalmente, a implantação desse monitoramento e fiscalização, os resultados atuais ainda são consideráveis. Portanto, um programa de monitoramento bem estruturado e métodos analíticos eficazes são importantes para a continuação desse programa de relevância para o país [4].

Além do programa da ANP, as próprias distribuidoras de combustíveis aplicam ações com o objetivo de eliminar ou desestimular ações ilícitas no setor de combustíveis. Entre elas, a aditivação de combustíveis por intermédio de substâncias marcadoras, que possibilitam a identificação do fornecedor (a bandeira da distribuidora de origem) e uma possível adulteração.

O objetivo desta prospecção foi obter as informações de patentes registradas relacionadas com marcadores de combustíveis líquidos automotivos.

\section{DESCRIÇÃO DA TECNOLOGIA}

Diversos tipos de aditivos são aplicados nos combustíveis automotivos com objetivos diversos; entre eles, os de identificação da distribuidora (Bandeira) e a possibilidade de identificação de adulteração.

Atualmente, o método utilizado oficialmente pela Agência Nacional do Petróleo, Gás Natural e Biocombustíveis, para a identificação de adulteração da gasolina, aplica a técnica de cromatografia gasosa para análise de marcadores que, se presentes nessa, constata a adulteração através de uso de solventes marcados (ANP, n. 274 2011). Esse método apresenta alguns aspectos desfavoráveis: 
a - cromatografia gasosa, que requer pessoal técnico especializado; como também, os equipamentos e insumos são caros, além da análise demandar um tempo relativamente grande e geralmente ser realizada apenas em laboratórios especializados;

b - o método identifica apenas alguns tipos de adulteração por solventes que são empregados comumente como adulterantes e por isso são marcados para possibilitar a identificação da adulteração.

Como visto, a identificação oficial da adulteração apresenta dificuldades devido à técnica empregada e a restritiva aplicação devido ao fato de apenas alguns solventes serem marcados e possíveis de identificação.

\section{METODOLOGIA}

A prospecção das patentes foi realizada no período do mês de outubro de 2011, utilizando a ferramenta de busca da worldwide.espacenet.com, da European Patent Office (EPO), empregando, inicialmente, algumas palavras chaves relacionadas com o tema marcadores de combustíveis; e, posteriormente, empregando os códigos de busca mais apropriados para o tema em questão. Aplicou-se a busca tanto usando o banco de dados da European Classification (ECLA), como também, da International Patent Classification (IPC). Também, foi feita a prospecção no banco de patentes do INPI (Instituto Nacional de Propriedade Industrial), em abril de 2012, considerando as palavras chaves marcadores e adulteração de combustíveis.

\section{RESULTADOS E DISCUSSÃO}

De acordo com a Metodologia e Escopo citados, empregou-se diversas palavras em português e inglês, utilizando as raízes destas (parte da palavra com asterisco: uma ferramenta de busca) para proporcionar um maior número de possibilidades para encontrar um maior número de códigos e patentes relacionadas ao tema. Como exemplo, usamos expressões como liquid* fuel*, fuel* mark*, adulter*, que representam palavras (ou raízes de palavras) como combustível líquido, marcador de combustível e adulteração, respectivamente, no banco de patentes da EPO. A seguir, apresentamos um resumo dos resultados na Tabela 1, para a pesquisa da EPO sendo que a última coluna da tabela representa o número de patentes registradas.

De acordo com o tema definido (marcadores de combustíveis) e o escopo, apresentado na Tabela1, definiu-se que o mais apropriado e mais abrangente foi realizar a prospecção a partir da conjunção dos códigos mais adequados que encontrou-se (marcados em vermelho e amarelo): C10L1 e G01N, Liquid carbonaceous fuels e INVESTIGATING OR ANALYSING MATERIALS BY DETERMINING THEIR CHEMICAL OR PHYSICAL PROPERTIES, respectivamente. Assim, com a conjunção desses dois códigos, associou-se o item combustível e as diversas formas e técnicas de investigação e análises desses materiais e de seus aditivos.

A partir desse quadro, importou-se os dados do banco da European Patent Office (EPO) e obteve-se uma planilha com um total de 243 patentes. O total de patentes importadas (243) é 
menor que o inicialmente indicado (716), pois o valor inicial está computado o total de registros de todas as patentes por famílias de patentes, o que acarreta, muitas vezes, em uma repetição do registro da patente.

Tabela1: Resultado da pesquisa no banco de dados da European Patent Office, através do emprego de raízes das palavras chaves relacionadas com o tema e os respectivos códigos relacionados (C10, G01N e os respectivos derivados).

\begin{tabular}{|c|c|c|c|c|c|c|c|c|}
\hline $\begin{array}{l}\text { Liquid* } \\
\text { fuel* }\end{array}$ & C10L & C10L1 & C10L1/00C & G01N & G01N23 & $\begin{array}{c}\text { fuel* or } \\
\text { Tag* or } \\
\text { label* or } \\
\text { mark* }\end{array}$ & Adulter* & $\begin{array}{l}\mathrm{EP}\left(\mathrm{N}^{\circ} \text { de }\right. \\
\text { patentes) }\end{array}$ \\
\hline \multirow[t]{15}{*}{$\mathrm{x}$} & & & & & & & & 64698 \\
\hline & $\mathrm{x}$ & & & & & & & 100000 \\
\hline & & $\mathrm{x}$ & & & & & & 100000 \\
\hline & & & $\mathrm{x}$ & & & & & 0 (IPC) \\
\hline & & & $\mathrm{x}$ & & & & & 1230 (EC) \\
\hline & & & & $\mathrm{x}$ & & & & 100000 \\
\hline & & & & & $\mathrm{x}$ & & & 63543 \\
\hline & & $\mathrm{x}$ & & & $\mathrm{x}$ & & & 4 \\
\hline & & $\mathrm{x}$ & & $\mathrm{x}$ & & & & 716 \\
\hline & & $\mathrm{x}$ & & $\mathrm{x}$ & & $\mathrm{X}$ & & 378 \\
\hline & & $\mathrm{x}$ & & $\mathrm{x}$ & & $\mathrm{x}$ & & 14 \\
\hline & & $\mathrm{x}$ & & $\mathrm{x}$ & & & & 16 \\
\hline & & $\mathrm{x}$ & & & & & $\mathrm{x}$ & 6 \\
\hline & & $\mathrm{x}$ & & $\mathrm{x}$ & & & $\mathrm{x}$ & 2 \\
\hline & & $\mathrm{x}$ & & & & & & 4 \\
\hline
\end{tabular}

Fonte: Autoria própria, 2012.

Na Figura 2, é apresentada a distribuição dos registros de patentes, por país, relacionadas com combustíveis líquidos e os métodos de análises de sua composição e dos aditivos, incluindo marcadores, considerando diversos aspectos.

Os Estados Unidos (US com 90 patentes), Japão (JP com 60), Alemanha (DE com 27) e França e Inglaterra (FR e GB, respectivamente, com 11) apresentam o maior número de patentes protocoladas, o que está diretamente relacionado com a aplicação e inovação de tecnologias relacionadas à qualidade dos combustíveis. A fundamental importância do controle da qualidade dos combustíveis na economia mundial promove a demanda por processos e métodos analíticos capazes de proporcionar garantias na qualidade do processo de produção e de comercialização desses combustíveis. Os países citados anteriormente são as sedes das principais empresas petrolíferas e de distribuição de combustíveis do mundo. Assim, justifica-se que esse países predominem no depósito desse tipo de patentes. 


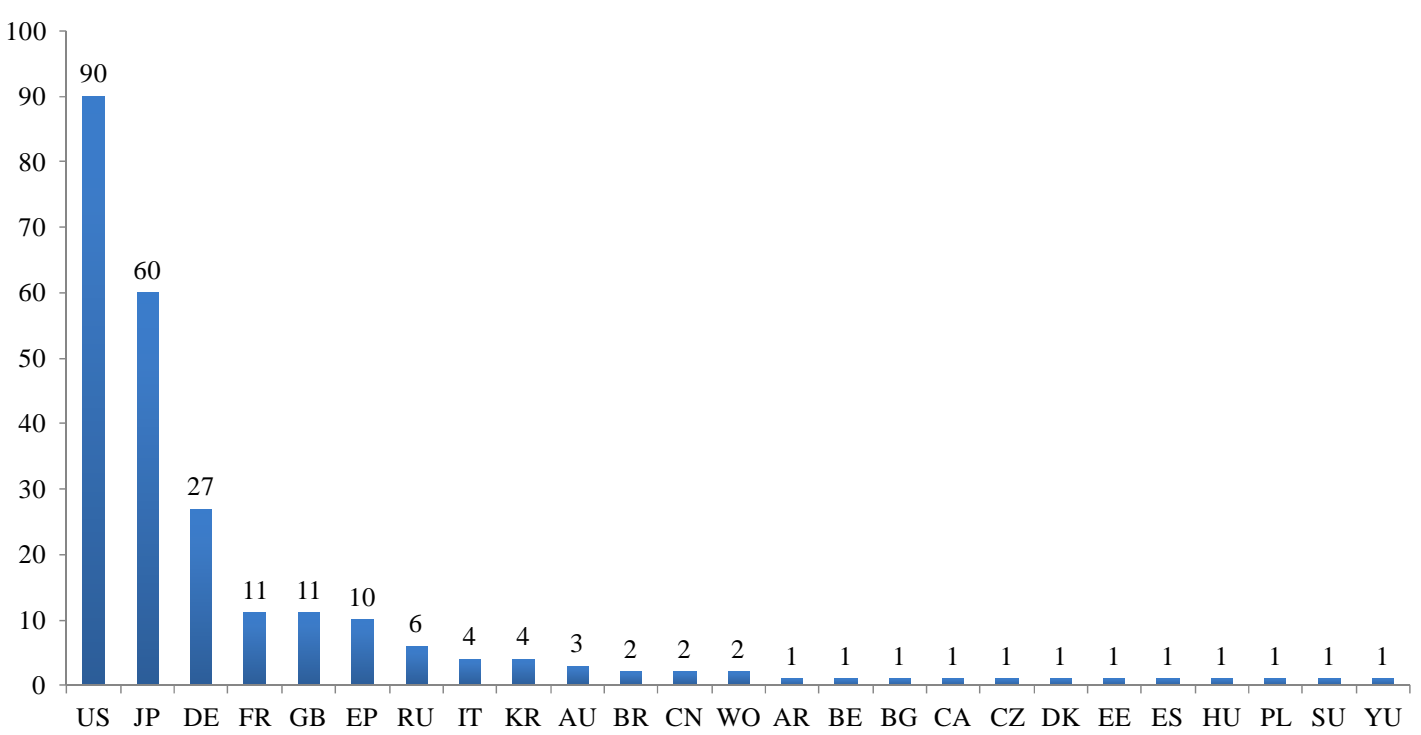

Figura 2: Número de patentes de combustíveis líquidos e métodos de análise de composição e de aditivos, incluindo marcadores, registradas por países na base da EPO. Fonte: Autoria própria, 2012.

O Brasil, nesse escopo e nessa base de dados da EPO, aparece com apenas duas patentes relacionadas com o tema, o que é muito pouco, considerando o desenvolvimento da nossa atual economia e o potencial que temos, já que possuímos uma das maiores empresas de exploração e processamento de petróleo do mundo. Podemos considerar que isso se deva a um relativo atraso no desenvolvimento de novas tecnologias, relacionadas ao tema adulteração de combustíveis e, principalmente, pela falta de uma cultura de depósitos de patentes, principalmente em nível internacional. Devemos considerar ainda que só viemos a ter um programa sistemático oficial de combate à adulteração a partir do ano 2000, através da ANP.

De acordo com a Figura 3, a seguir, percebe-se que houve um aumento no número de registros de patentes a partir do ano de 1980, mantendo-se certa regularidade por duas décadas, e uma indicação de queda a partir de 2008. Esse aumento pode estar relacionado com a grande demanda impulsionada pelo avanço da economia mundial, suportada basicamente pela indústria petrolífera, o que exigiu uma maior produção e uma melhor qualidade dos combustíveis, considerando, também, o início de uma maior preocupação com os fatores ambientais da produção e consumo (KALLIGEROS, 2003). Por meados de 2008, percebe-se uma provável tendência de redução do número de patentes registradas relacionadas com o tema da prospeç̧ão. Por ser um tema já bastante explorado (vide o número de patentes relacionadas, Tabela1) e por, na última década, se intensificar a ideia de que é necessária uma redução progressiva das emissões gasosas provenientes da queima de combustíveis fósseis, com intuito de redução do impacto ambiental e, por consequência, a procura de fontes renováveis alternativas de energia, justifique essa atual tendência de redução do número de patentes relacionadas a análise e composição de combustíveis automotivos, ou seja, uma certa saturação do tema. 
Na Figura 4, a seguir, apresenta-se o número de patentes por principais autores, fazendo um corte a partir de um mínimo de três patentes depositadas. Verifica-se uma maior predominância de autores alemães, sendo o destaque o autor Beck K.H com 15 patentes.

Na Figura 5, apresenta-se o número de patentes depositadas pelas principais empresas, considerando um mínimo de depósitos de três patentes.

Seguindo a lógica do que vimos na distribuição por países (Figura 2), as principais companhias químicas, petroquímicas e petrolíferas dos países desenvolvidos são responsáveis pelo maior número de depósitos desse tipo de patentes, o que também está relacionado com a cultura de inovação tecnológica e de propriedade industrial desses países.

Na Figura 6, são apresentados os diversos tipos de aplicações relacionadas com as patentes prospectadas.

De acordo com a figura anterior, observa-se os diversos tipos de aplicações relacionadas com análises de combustíveis e seus marcadores. Dessas, cerca de metade das aplicações (46\%) está relacionada diretamente com marcadores, o que demonstra a importante aplicação desses tipos de moléculas, principalmente em termos do controle da qualidade de combustíveis. Esse tipo de aplicação vem se tornando uma importante ferramenta no combate a adulteração, se difundindo em todo mundo (KALLIGEROS, 2001; MOHAMMAD, 2008).

Por fim, a Figura 7 apresenta o número de patentes depositas no INPI, relacionadas com o tema. A distribuição das patentes, num total de 31, foi relacionada com a aplicação de novos equipamentos e dispositivos, desenvolvimento de marcadores ou desenvolvimento de novas técnicas ou aplicações. Apesar de um número razoável de patentes, considerando a nossa cultura em termos de propriedade industrial, esse campo ainda é bastante fértil para desenvolvimento de novas patentes relacionadas ao tema, visto que os índices de adulteração ainda são relativamente altos no país.

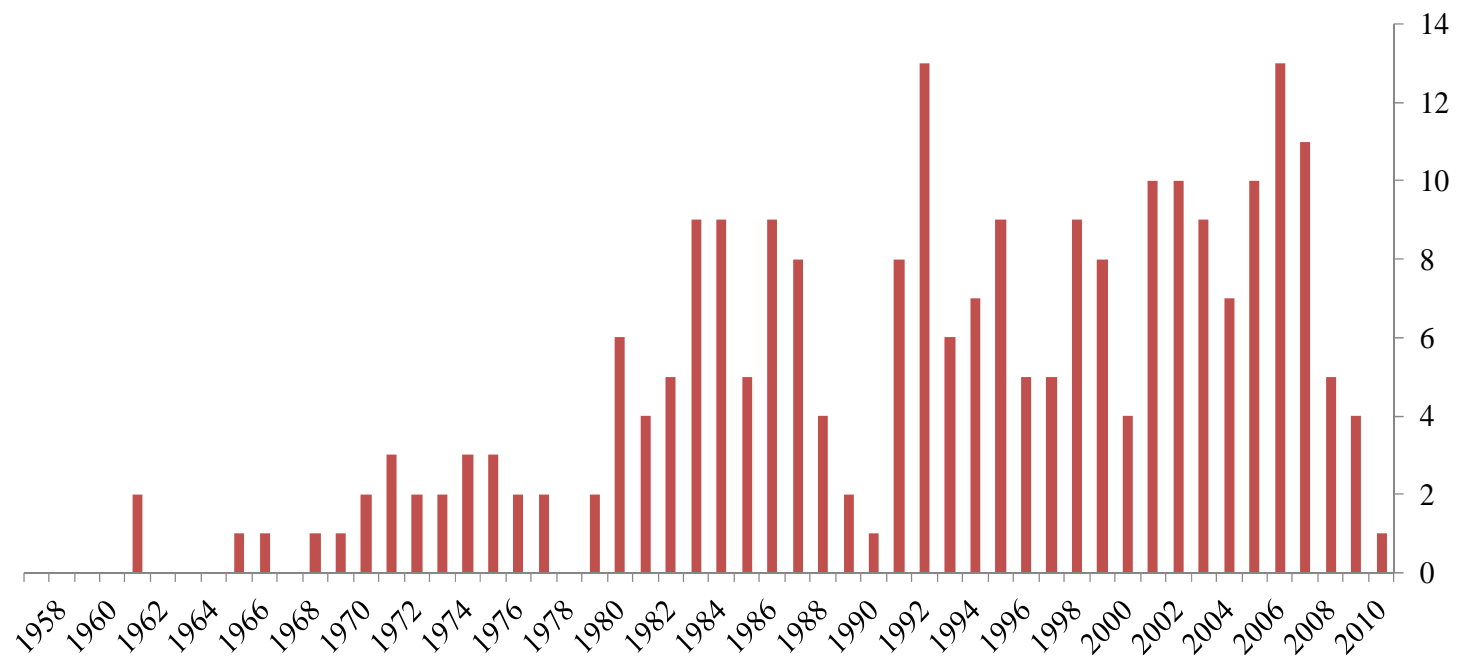

Figura 3: Número de patentes de combustíveis líquidos e métodos de análise de composição registrados por ano - base EPO. Fonte: Autoria própria, 2012. 


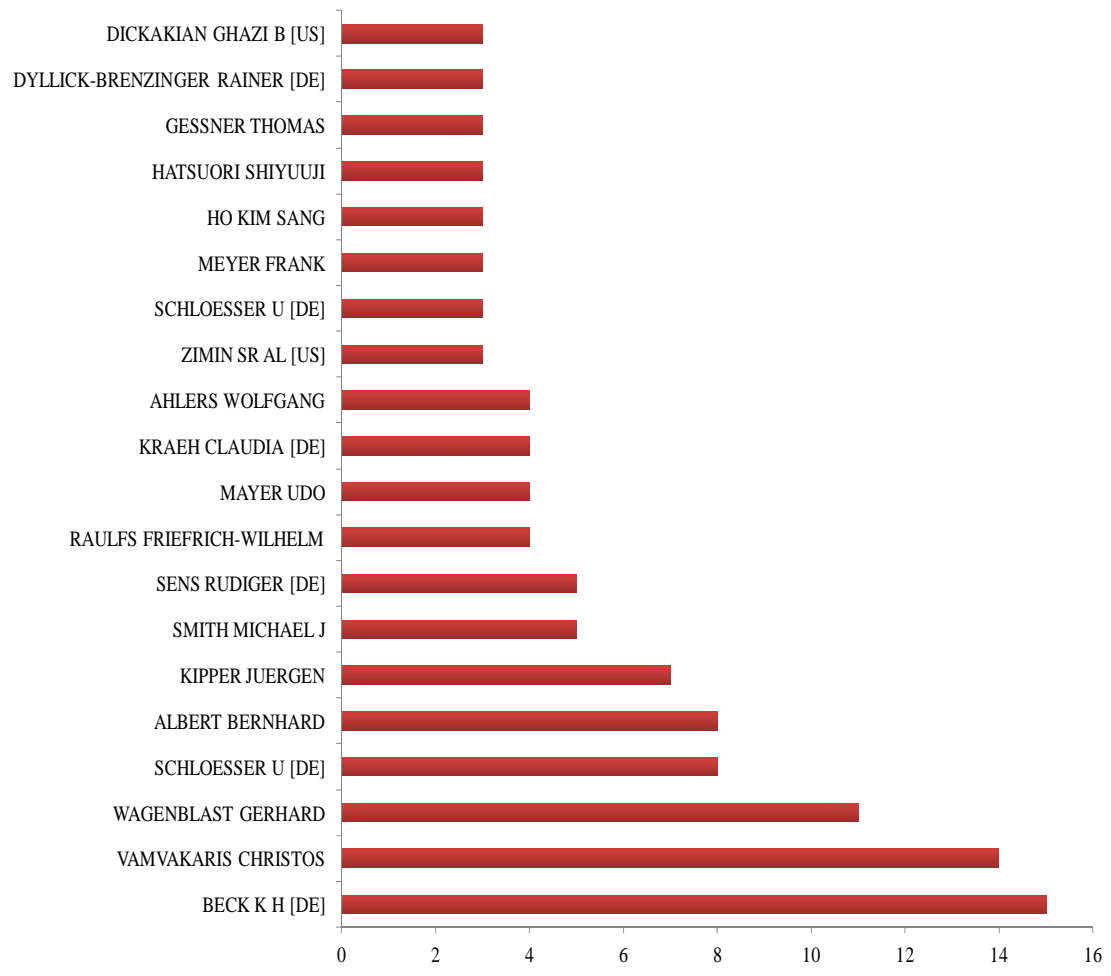

Figura 4: Gráfico do número de patentes depositadas por principais autores, considerando a partir de três patentes depositadas - base EPO. Fonte: Autoria própria, 2012.

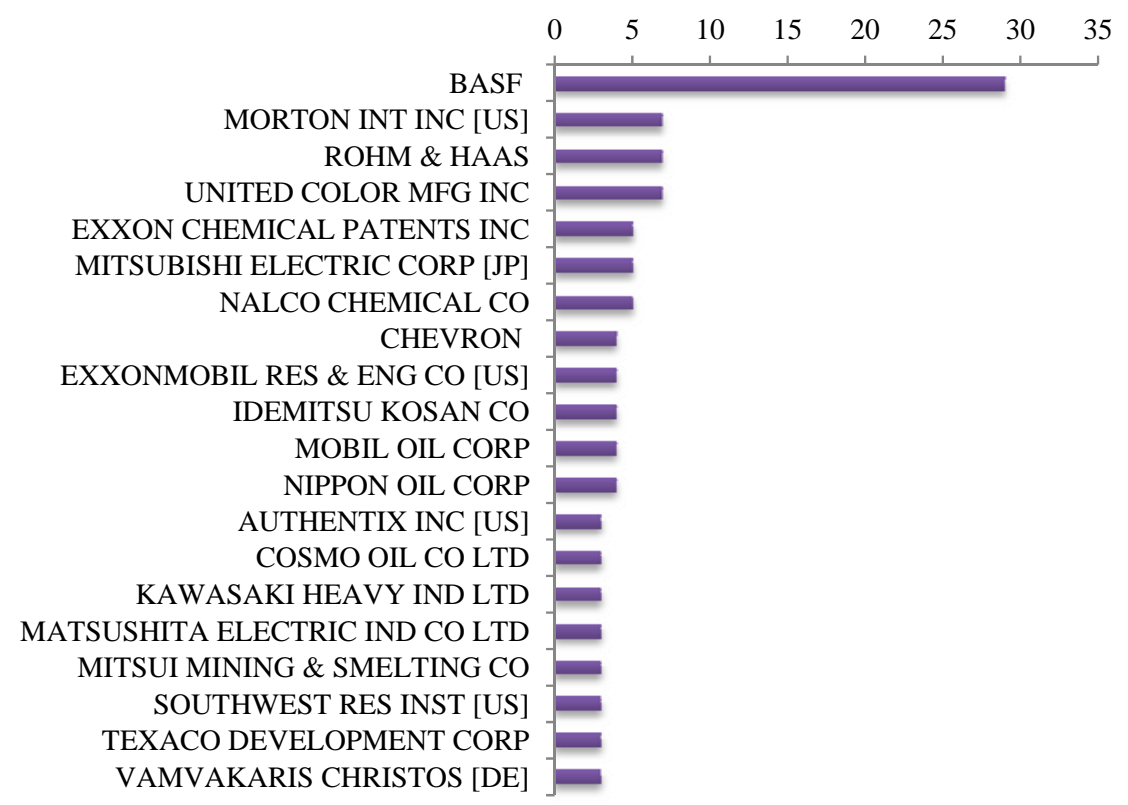

Figura 5: Número de patentes depositadas por empresas, considerando um mínimo de três patentes - base EPO. Fonte: Autoria própria, 2012. 


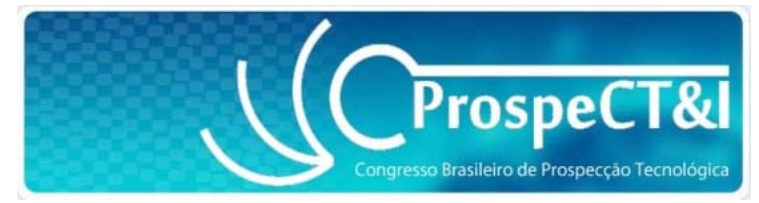

Marcador fluorescentes combustíveis Marcador combustíveis

Marcador fluorescente líquidos

Macador p/ líquidos

Marcador, petrolífero, orgânicos

Método analítico combustíveis

Método de análise

Aditivos, moléculas

Produção, composição combustível

Combustão

Equipamentos

Mistura, fluxo

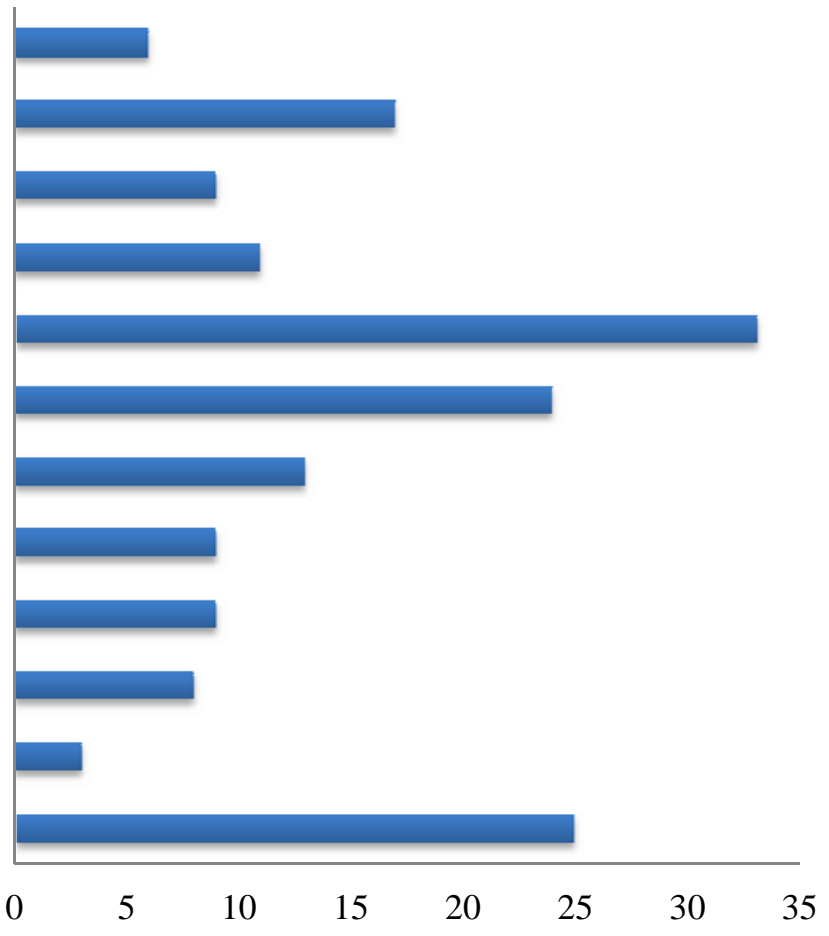

Figura 6: Porcentagens das diversas aplicações das patentes, considerando o total de patentes relacionadas com a análises de petrolíferos e combustíveis e respectivos marcadores - base EPO. Fonte: Autoria própria, 2012.

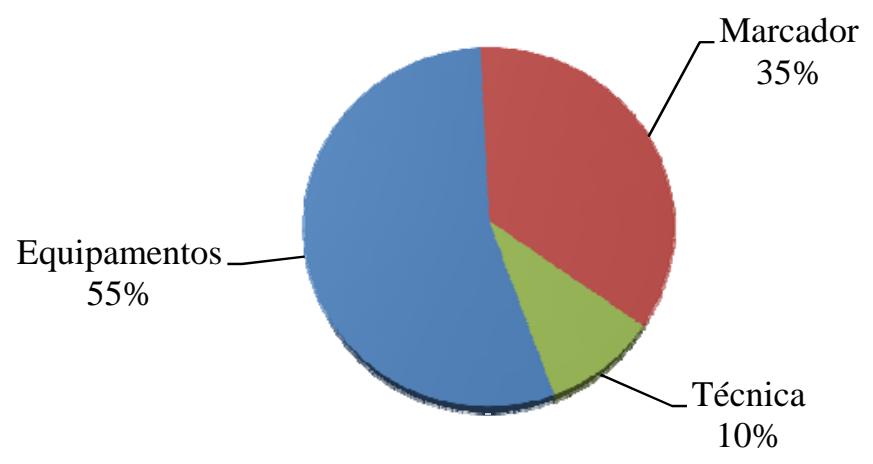

Figura 7: Número de patentes depositadas no INPI relacionadas com o tema combustíveis e adulteração. Fonte: Autoria própria, 2012. 


\section{CONCLUSÃO}

Em todo o mundo, o problema da adulteração dos combustíveis ainda é muito impactante financeiramente para o consumidor e para a economia do país, além de poder estar associado com o crime organizado. Apesar das nações apontarem para utilização de novas formas de energia e, consequentemente, combustíveis menos poluentes, os combustíveis fósseis ainda são a base das grandes economias mundiais. Não é á toa que os países desenvolvem esforços inmensuráveis na procura de novas fontes e bacias de petróleo em todo mundo; como exemplo, o próprio Brasil que desenvolveu uma importante tecnologia de prospecção de petróleo a grandes profundidades (região do pré-sal) em alto mar.

Como foi visto nos gráficos anteriores, no mundo inteiro, há uma preocupação com o desenvolvimento de novas tecnologias no controle de qualidade dos combustíveis, em especial com a adulteração desses. A análise ou investigação da qualidade dos combustíveis é um processo que demanda logística, técnicos capacitados, laboratórios acreditados ou qualificados, metodologias e técnicas analíticas, geralmente, complexas, demoradas e caras.

Portanto, no Brasil, país emergente e com a perspectiva de aumentar cada vez mais a produção desses tipos de combustíveis fósseis e, por também, possuir dimensões continentais, dificultando a fiscalização, a adulteração de combustíveis se apresenta como um problema concreto e impactante. Assim, a aplicação de marcadores eficientes de combustíveis aliada a técnicas de análises mais simples e baratas poderá proporcionar métodos mais eficazes contra o problema disseminado da adulteração de combustíveis no país.

\section{PERSPECTIVAS}

O desenvolvimento de marcadores eficientes para combustíveis líquidos que apresentem também outras características vantajosas para os combustíveis, associados a técnicas analíticas mais baratas, robustas e rápidas para a identificação de adulteração, apresenta-se como uma fonte promissora de inovação tecnológica. Apesar de já termos algumas patentes na área (vide Figura 7), ainda é um campo para desenvolvimento de novas patentes. A própria ANP (ANP n. 13, 2009) possibilita o desenvolvimento de novos marcadores, que prevê o cadastramento de empresas interessadas em fornecê-los, o que ajudaria a impulsionar o conceito de propriedade intelectual e inovação tecnológica no Brasil.

\section{REFERÊNCIAS}

AGÊNCIA NACIONAL DO PETRÓLEO (ANP). Gás natural e biocombustíveis. Brasília. Portaria ANP n ${ }^{0} 248$ de 31 de out. de 2000.

AGÊNCIA NACIONAL DO PETRÓLEO (ANP). Gás natural e biocombustíveis - Portaria ANP n ${ }^{0} 274$ de 1/11/2011 (DOU de 5/11/2001).

AGÊNCIA NACIONAL DO PETRÓLEO (ANP). Gás natural e biocombustíveis Resolução ANP nº 13/2009.

AGÊNCIA NACIONAL DO PETRÓLEO. Gás natural e biocombustíveis. Brasília. Portaria de ${ }^{0} 311$ de 27 de dezembro de 2000. 
KALligEROS, F. ZANNIKOS, S. STOURNAS, E. LOIS and G. Anastopoulos. Fuel adulteration issues in Greece. Energy, v. 28, n. 1, p. 15-26, 2003.

KALLIGEROS, S.; ZANNIKOS, F.; STOURNAS, S.; LOIS, E.; ANASTOPOULOS, G. A survey of the automotive diesel quality in the Athens. Energy. v. 25, p. 1381-1390, 2001.

MOHAMMAD A.; Al-GHOUTIA; YAHYA S.; Al-DEGSB. Mohammad Amer. Determination of motor gasoline adulteration using FTIR spectroscopy and multivariate calibration. Talanta, v. 76, p. 1105-1112, 2008.

PMQC - Programa de Monitoramento da Qualidade dos Combustíveis Líquidos. Disponível em: <www.anp.gov.br>. Acessado em: 00.00. 00.

WIEDMANN, L. S. Brazilian gasoline quality: Study of adulteration by statistical analysis and gas chromatography. Journal of the Brazilian Chemical Society. v. 16, n. 2, p. 139146, 2005. 